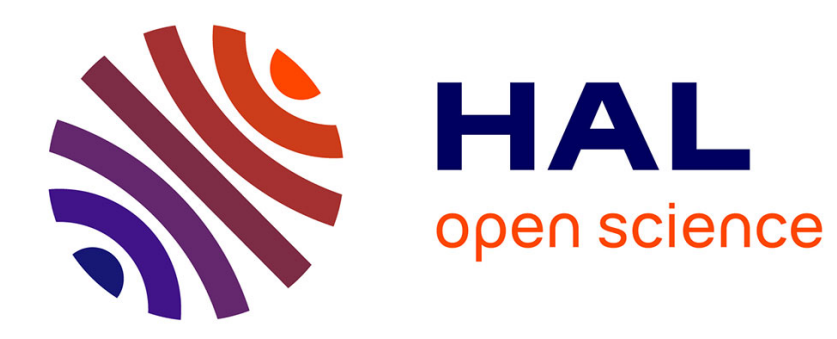

\title{
Souffrances des pères lors des divorces
}

Claire Metz

\section{To cite this version:}

Claire Metz. Souffrances des pères lors des divorces. Neuropsychiatrie de l'Enfance et de l'Adolescence, 2005, 53 (5), pp.238-244. 10.1016/j.neurenf.2005.09.005 . hal-03049614

\section{HAL Id: hal-03049614 https://hal.science/hal-03049614}

Submitted on 9 Dec 2020

HAL is a multi-disciplinary open access archive for the deposit and dissemination of scientific research documents, whether they are published or not. The documents may come from teaching and research institutions in France or abroad, or from public or private research centers.
L'archive ouverte pluridisciplinaire HAL, est destinée au dépôt et à la diffusion de documents scientifiques de niveau recherche, publiés ou non, émanant des établissements d'enseignement et de recherche français ou étrangers, des laboratoires publics ou privés. 


\title{
Souffrances des pères lors des divorces \\ Sufferings of the fathers in divorces
}

\author{
C. Gaudard-Metz \\ Psychanalyste, psychologue clinicienne, URPCLS-EA 3071, Université Louis-Pasteur, 67000 Strasbourg, 15, allée de la Robertsau, \\ 67000 Strasbourg, France
}

Reçu le 25 février 2005 ; accepté le 13 septembre 2005

\begin{abstract}
Résumé
Sous la pression des transformations des formes de la famille, des évolutions du droit, et des avancées des sciences biologiques et médicales, les repères institutionnels de la paternité ont été bouleversés. Auparavant, le père était désigné par les liens du mariage et était pourvu de la puissance paternelle. La mort du paterfamilias dans le droit et le déclin du mariage ont bouleversé ces fondements. Les pères font désormais l'objet de soupçons. De plus, la société a organisé une véritable éviction juridique des pères dans le cadre des divorces. Or, devenir père nécessite un travail subjectif, qui s'appuie sur un temps politique lié aux montages juridiques et institutionnels. La défaillance de ce système dans la période contemporaine a pu rendre ce travail difficile pour chaque père divorcé. Nos exemples cliniques montrent la résonance entre les souffrances singulières des pères et leur exclusion juridique. Mais ils montrent aussi les facteurs singuliers conscients et inconscients qui ne proviennent pas forcément du divorce.
\end{abstract}

\begin{abstract}
Under pressure from the transformations of the forms of family, from the evolutions of law, and from the advances of the biological and medical sciences, the institutional points of reference of the fatherhood have been changed. Before, the father was designated by the bonds of marriage and endowed with paternal power. The suppression of paterfamilias in the law and the decline of marriage changed these foundations. From then on, the fathers are the object of suspicions. Furthermore, society has organised a real legal eviction of the fathers in the framework of divorces. Now, becoming the father requires a subjective work, which depends on a political time related to legal and institutional set-ups. The default of this system, during the contemporary period could have made this work difficult for each divorced father. Our clinical examples show the echo with singular sufferings of the fathers and their legal exclusion. But they also show the singular conscious and unconscious factors, which do not necessarily come from divorce.
\end{abstract}

Mots clés : Divorce ; Père ; Souffrance ; Droit ; Inconscient

Keywords: Divorce; Father; Suffering; Law; Unconscious

Depuis les années 1970, l'institution familiale a subi des bouleversements sans doute irréversibles. Trois sortes de facteurs sont à distinguer : les transformations pour ne pas dire les mutations des formes de la famille - le divorce en est une cause et il est devenu un fait de société —, les avancées

Adresse e-mail : claire.metz@voila.fr(C. Gaudard-Metz). des sciences biomédicales, et les évolutions du droit qui accompagnent ou répondent plus ou moins efficacement à ces changements.

Sous cette triple poussée, les fondements de l'« institution du père » ont été bouleversés, et les pères contemporains, en particulier les pères divorcés, ne sont pas sans en avoir subi des effets. Psychologue au centre médicopsycho-pédagogique (CMPP), et psychanalyste, j'ai pu écouter certains de ces 

pères, venus consulter pour leur enfant, exprimer leurs propres souffrances lors des divorces.

\section{Perte de repères}

Géraldine neuf ans, est amenée en consultation par sa mère, car elle manifeste depuis peu un ensemble de symptômes : des troubles anxiophobiques, un sommeil perturbé, des troubles anorexiques, un besoin permanent de la présence maternelle, elle dort dans le lit de la mère et du beau-père. En exposant ces difficultés, Mme G. m'interroge : "Peut-être souhaitez-vous voir le papa et lequel ? »

Cette question est bien révélatrice d'une époque qui a perdu les repères de la paternité (Bruel, 1998) [1], si bien que nous ne savons plus ce qu'est un père, ni même qui est le père : est-ce le père éducateur, le géniteur, le père légal ? (Hurstel, 2002) [2]. Dans ce cadre général de flottement au sujet du père, l'inquiétude s'est focalisée sur les pères divorcés ou séparés et sur l'importance de la disparition des liens entre le père et ses enfants lors des divorces, révélée par une enquête démographique effectuée en 1994 (Villeneuve-Gokalp, 1999) [3]. Quels sens et quels effets attribuer à ces ruptures de lien?

Un certain discours, éducatif, pédagogique ou médiatique depuis les années 1950 environ, tend à faire porter à l'absence du père de famille la responsabilité de tous les maux dont souffre la société : toxicomanie, addictions en tous genres, échec scolaire... Or, cette question se révèle largement plus complexe et nous pouvons affirmer que tout dépend du sens que chacun des membres du groupe familial accorde à cette absence (Hurstel, Hoffmann, 1993), (Metz,1999) [4,5]. Cependant, les pères divorcés souffrent de ces représentations négatives sur le père absent. Ils sont certes loin d'être tous en situation de rupture vis-à-vis de leurs enfants et certains pères divorcés se sont constitués en associations plus ou moins revendicatives (Chombeau, 1993) [6].

Dans ce malaise généralisé, cette floraison de questionnements et de discours portés sur les pères ou sur la question du père, en particulier du père divorcé, nous nous sommes intéressés à la parole de parents venus consulter en CMPP pour leur enfant lors d'un divorce, en reprenant les notes prises lors des séances, dans l'après-coup de ces consultations, c'està-dire lorsque les séances avaient pris fin. Nous exposerons deux exemples cliniques, ceux de Géraldine et de Bertrand. Si chaque cas reste toujours singulier, nous les avons choisis car ils nous paraissent représentatifs des difficultés exprimées couramment par les pères que nous recevons dans ces consultations apparemment provoquées par un divorce, et qui se multiplient au sein du CMPP où j'exerce.

Auparavant, afin de préciser le contexte qui entoure les pères divorcés - et nous montrerons qu'il n'est pas sans lien avec ce dont ils souffrent —, nous définirons les modalités contemporaines des bouleversements de "l'institution du père $\gg$.

\section{L'institution du père}

\subsection{La fin de la puissance paternelle}

Les fondements du statut juridique du père dans notre code civil s'enracinent dans le droit romain. La paternité romaine découle d'une adoption, c'est un produit de la loi qui signifie qu'être géniteur [genitor] ne suffit pas en soi pour accéder au titre de père [pater]. « Le fondement juridique de la paternité réside ainsi dans la volonté d'un homme de se constituer père » (Delumeau, Roche, dir., 2000, 44) [7]. C'est pourquoi nous parlons d'institution du père, qui est une construction juridique et culturelle possédant deux caractéristiques :

- l'importance accordée à l'institution du mariage qui noue toutes les fonctions du père dans la personne du mari. Le père est « celui que le mariage désigne » selon la célèbre formule du code civil de 1804. Le lien de paternité est indissociable du lien conjugal dont il découle ;

- le père en tire un regain d'autorité, il apparaît comme le garant de l'ordre public et le pilier de la société qui devient patriarcale, jusqu'au Prince « père de ses sujets » (Delumeau, Roche, dir., 2000, 41) [7]. C'est l'« âge d'or» du père, qui va de la renaissance jusqu'à l'époque des lumières, et qui imprègne toujours notre imaginaire. À la fin de l'ancien régime, « le seul père est le père de famille, à la fois géniteur, éducateur et vecteur du patrimoine et du nom. » (Delumeau, Roche, dir., 2000, 291) [7].

Nous saisissons quel profond bouleversement des repères s'est produit lorsqu'en 1972 le nombre des mariages commence à s'effondrer, entraînant avec lui toute cette construction vieille de plusieurs siècles. La loi de 1970, abolissant la puissance paternelle au profit de l'autorité parentale, signe la mort du paterfamilias dans le droit.

Pourtant, notre imaginaire collectif reste imprégné par des références issues de cette période historique révolue : le «père fort » et le « père carent ». Le « père fort » est un héritage du paterfamilias et face à ce père héroïque, un père de famille n'est jamais vraiment à la hauteur, c'est un « pauvre type » (Lacan, 1994, 230) [8]. Ainsi, se constitue la deuxième figure issue de notre imaginaire collectif, le « père carent », dont le père absent est un avatar. Ces deux figures étroitement liées entre elles comme l'avers et le revers d'une médaille, persistent avec autant d'insistance à cause du basculement des repères de la paternité dans les 30 dernières années. Faute de repères, les figures du « père à poigne » et du « mauvais père » sont apparues, à tort, comme des valeurs de référence.

De plus, à partir du xix ${ }^{\mathrm{e}}$ siècle, les pères font l'objet de contrôle de la part de l'état et des spécialistes, et ils ne vont cesser d'être soupçonnés, cela jusqu'aux accusations contemporaines d'inceste dans les jugements de divorce. Ainsi, les pères, en particulier les pères divorcés, souffrent de représentations collectives imaginaires historiquement dépassées, et d'endosser malgré eux le « pardessus du soupçon ». (Hurstel, Delaisi de Parceval, 2000, 381) [9]. Au poids de ces représentations dévalorisantes vont s'ajouter des difficultés issues des inadéquations du droit face aux transformations des formes familiales. 


\subsection{Les transformations des formes de la famille}

À partir de 1972 le nombre des divorces s'accroît brusquement, tandis que celui des mariages décroît inexorablement. La quantité de concubinages ne cesse d'augmenter ainsi que la proportion d'enfants naturels, au point de devenir actuellement le mode presque habituel de constitution du couple (Beaumel et al., 1999) [10].

\subsection{Les inadéquations du droit entre 1970 et 2002}

\subsubsection{Filiation et autorité parentale}

En 1970, dans un premier temps, l'exercice de l'autorité parentale était préférentiellement accordé à la mère seule dans les familles naturelles. En effet, les mères reconnaissaient les enfants en plus grand nombre à ce moment-là, et obtenaient dans ce cas l'autorité parentale. Dans le cas d'une double reconnaissance, elles l'avaient également automatiquement. Le père devait faire une démarche supplémentaire s'il désirait l'exercice de l'autorité parentale. Les lois évoluent ensuite progressivement, mais ce n'est qu'en 2002 que le législateur établira la responsabilité parentale de manière identique pour les parents mariés, pour les parents séparés ou divorcés, et les parents ayant choisi toute forme d'union ou s'étant séparés après une union, que ces parents soient naturels ou légitimes. (Loi ordinaire 2002-305).

Auparavant, dans les familles divorcées ou séparées, le parent qui a « la garde » de l'enfant (terme utilisé jusqu'en 1987), dans la majorité des cas la mère, obtenait seule l'autorité parentale. Nulle part l'idée que les enfants appartiennent aux mères n'est plus apparente que dans les attributions de « garde» (Villeneuve-Gokalp, 1993, 68) [12]². Cette idée est partagée par les femmes et les hommes : car si les tribunaux accordent préférentiellement la garde d'enfants en bas âge aux mères, les pères demandent rarement la garde de leurs enfants.

En 1970 les familles non maritales étaient encore une minorité. Mettre l'enfant sous l'autorité exclusive de la mère relevait alors d'une mesure destinée à assurer le bien de l'enfant. Mais le législateur de 1970 n'avait pas prévu l'accroissement brutal du nombre de ces familles et par-là, des pères évincés de leurs droits. Pour toutes ces raisons, F. Hurstel concluait en 1991 que « dans les familles non maritales le droit des mères est tel que l'on peut parler d'une " exclusion juridique » du père » (Hurstel, 1991, 218) [11], dont ont souffert les pères divorcés.

Il faut ajouter, pour bien comprendre cette exclusion juridique des pères, deux éléments : une certaine méconnais-

\footnotetext{
${ }^{1}$ Le nombre de mariages décroît entre 1971 et 1982 , on passe de 400000 mariages par an à 312000 . Le nombre de divorces augmente, il double de 1970 à 1980 (Hurstel, 1991, 214)[11] De $6 \%$ en 1966, la proportion d'enfants naturels passe à un enfant sur sept en 1982 .

${ }^{2}$ Entre 1972 et 1985 , la garde est attribuée à la mère dans $85 \%$ des cas, au père dans $9 \%$ des cas, aux deux parents dans $5 \%$ des cas, et à un tiers dans $1 \%$ des cas (Villeneuve-Gokalp 1993, 68) [12].
}

sance de la loi de la part des pères, et le temps que nécessitera l'évolution des mentalités. Dans un premier temps après 1972, les pères dans les familles naturelles, se sont peu préoccupés de l'autorité parentale, dont ils ignoraient les modalités d'accès. Un grand nombre d'entre eux ne reconnaissaient pas leurs enfants. Il faudra plusieurs décennies pour que les pères non mariés effectuent quasi automatiquement cette démarche à la naissance de leur enfant ${ }^{3}$. (Beaumel et al., 1999), (Doisneau, 2002) [10,13].

\subsubsection{Critères de désignation du père}

Le système de filiation a été de tout temps une construction juridique et culturelle, et ne saurait se réduire au lien biologique, « conception bouchère de la filiation » (Legendre, 1989, 171) [14]. Si le problème est partout le même : «il y a d'une part un homme et de l'autre un nouveau-né, les solutions techniques pour rattacher le second au premier peuvent être fort différentes » (Delumeau, Roche, Dir., 2000, 43) [7]. Forcément abstraites, ces solutions ne peuvent procéder que d'une fiction juridique. Pendant plusieurs siècles, la «présomption de paternité » désignait sans équivoque les pères par le biais du mariage qui nouait en un seul homme, le père, toutes les fonctions paternelles. Trois ordres de faits renversent l'évidence à partir de 1972 : le déclin du mariage, les nouveaux critères de désignation des pères et les avancées des sciences biomédicales.

\subsubsection{La disjonction entre le lien de filiation et le lien conjugal}

Auparavant, la présomption de paternité unissait les trois personnes du père, de la mère et de l'enfant. Or, en égalisant les filiations de l'enfant naturel et de l'enfant légitime, la loi du 3 janvier 1972 met en avant les liens du sang au détriment de la présomption de paternité qui s'affaiblit. Cette loi opère une disjonction inédite entre le lien conjugal et le rapport vertical entre père et enfant. Dans les familles naturelles, le lien de paternité ne dépend plus du lien entre père et mère.

\subsubsection{La suprématie du critère biologique pour désigner le père}

En 1972, le législateur ne prévoyait pas que la paternité allait devenir démontrable au moyen du test d'ADN. Dans la jurisprudence, le critère de vérité biologique va prendre une ampleur imprévue et le père est bien vite assimilé au géniteur. Les critères de désignation des pères : « vérité biologique » et " possession d'état» au lieu de garantir par différents moyens un père à l'enfant, vont au contraire se concurrencer du fait de la prépondérance accordée au critère biologique, provoquant ainsi dans certains cas une absence

\footnotetext{
${ }^{3}$ Alors qu'au début des années 1970, un enfant sur cinq (20\%) était reconnu à la naissance par son père dans les familles hors mariage, ce sont en 1996 les trois-quarts $(75 \%)$ des enfants nés hors mariage qui sont reconnus par leur père à la naissance. En 25 ans, entre 1975 et 1990, la proportion d'enfants non reconnus par leur père cinq ans après la naissance a baissé de moitié (de 25 à $12 \%$ ).
} 
de filiation paternelle. Les procréations médicalement assistées vont mettre l'accent sur le problème posé par la vérité biologique comme critère dominant pour désigner le père.

Ainsi, à partir de 1970, en trois décennies, l'institution du père a été profondément bousculée dans ses fondements, c'est pourquoi Pierre Legendre peut évoquer « la raréfaction du père dans nos sociétés » (Legendre, 1989, 171) [14]. En 1970, le législateur prenait surtout acte de l'évolution du statut des femmes, et égalisait dans le mariage les statuts du père et de la mère. Par la suite, l'inadéquation du droit face aux modifications des formes de la famille a provoqué, comme nous l'avons montré, une véritable éviction légale des pères.

La société se réveille avec de mauvaises surprises : les filiations paternelles sont instables, certains pères divorcés ou séparés de leur compagne s'absentent de la vie de leurs enfants jusqu'à disparaître parfois définitivement. Les rapports alarmistes de spécialistes se succèdent. Quels liens s'opèrent-ils entre cette institution du père déconstruite et les hommes devenus pères lors de cette période, ainsi que les souffrances dont font état les pères divorcés?

\section{Devenir père}

Le sujet devient père en renonçant à sa condition de fils pour la garantir à son propre fils, par une opération de « permutation symbolique » : il échange la place de fils contre celle de père (Legendre, 1985, 301) [15]. Il n'accumule pas les places mais perd celle de fils, le père doit abandonner sa propre condition d'enfant pour la laisser à son enfant. Cela ne va pas de soi et constitue un véritable travail subjectif, qui n'est possible que si auparavant, à la génération précédente, son propre père lui ait déjà cédé sa place d'enfant. Pour être père, quelque chose doit mourir dans le sujet, qui touche sa représentation identificatoire à son propre père et ce passage peut être interprété comme meurtre du père (Legendre, 1985, 307) [15].

Cette perte subjective permet d'éviter une confusion générationnelle où le sujet devenu père pourrait se croire inconsciemment père de son propre père, s'il ne cédait justement cette place de fils. Cette confusion entre pères (le père de l'enfant et le père du père), qui entraînerait une logique de duel où chacun serait réciproquement amené à se représenter le meurtre du père, n'est évitée que par un «forçage juridique » (Legendre, 1985, 303) [15]. Les montages institutionnels articulent chaque place généalogique de telle manière que juridiquement aucune confusion ne soit possible.

Ainsi, l'écart généalogique entre le fils devenant père et son propre père se fabrique en deux temps : le temps familial du travail subjectif, éminemment lié à l'œdipe puisque sa sortie conditionne « la juste situation du sujet par rapport à la fonction de père » (Lacan, 1994, 204) [8]. Le deuxième temps est un temps politique, institutionnel, car il se réfère aux textes et à la légalité paternelle.

La permutation est dite symbolique car le sujet rencontre « le discours qui fait loi pour l'espèce » (Legendre, 1985,
303) [15]. C'est ce discours qui institue le père comme fonction dans le domaine social, et qui fait que chaque père ne fait que représenter la fonction. La problématique père-fils échappe donc au cadre strictement familial. L'inverse produirait des pères souverains au sein des familles, le père du caporal Lortie en est un exemple (Legendre, 1989) [14]. Lacan nous a mis en garde contre les effets destructeurs de la figure paternelle dans le cas des pères s'arrogeant les fonctions de législateur, tel que le père du Président Schreber. La fonction paternelle vient donc nécessairement s'enraciner dans l'ensemble des systèmes institutionnels que l'humanité met en place pour se survivre et se perpétuer. Ainsi, le dispositif de la filiation fonctionne selon deux temps qui sont à différencier. Le temps familial ne saurait se passer du temps politique de l'institution du père, instaurée par les montages généalogiques, et qui fonctionne comme véritable tiers social. En conséquence, la fragilisation de l'institution du père peut venir directement ébranler la fonction paternelle.

Les sujets sont les fruits de leur histoire personnelle mais aussi de l'histoire collective et des institutions (Thévenot, 1993)[16]. Les hommes devenus pères après 1970 ont subi les bouleversements institutionnels de la paternité que nous avons décrits. Comment ces hommes, ont-ils « fait affaire » (Hurstel, 1994, 74) [17] avec ces transformations, dans le cadre des divorces et des séparations?

\section{Le temps familial, un temps subjectif}

Selon Lacan le lien de paternité n'est pas premier mais il est second car il est subordonné à l'orientation première du désir du père envers la mère (Lacan, 1974-1975, 21 janvier 1975) [18] et de la mère envers le père. De cela nous déduisons que pour étudier la souffrance des pères de famille, il nous faut examiner les enjeux psychiques en cause tant pour le père que pour la mère et l'enfant. Cette perspective nous a permis de dégager certains enjeux qui seraient restés sans cela masqués, et que nous illustrons dans nos exemples cliniques à travers les cas de Géraldine et Bertrand.

Dans ces exemples, nous avons extrait du corpus de données discursives que constituaient les notes prises lors des séances, certaines des paroles des personnes reçues, présentées sous forme de citations. Bien qu'il s'agisse bien sûr toujours d'extraits, nous souhaitons de cette manière rester au plus près de la parole des sujets eux-mêmes.

\section{Exemples cliniques}

\subsection{Une parole nulle et non avenue : le père de Géraldine}

Le père explique : « Géraldine est assez renfermée, assez coincée. Elle est sous l'emprise de sa mère » Il ajoute que sa mère « l'étouffe, même quand elle est avec moi ». À l'appui, il précise qu' « elle ne met pas les habits si sa mère ne lui permet pas » et que « sa mère c'est la parole du bon Dieu ». Il 
déplore que sa fille de neuf ans ne se prenne pas plus en charge elle-même. «Qu'est-ce qu'elle fait d'elle-même ? Pas grandchose » Il reproche à son ex-épouse de « lui couper la viande, tu lui fais tout le temps ». La mère s'en défend puis s'en justifie car « Géraldine aime la facilité, elle aime que ça lui tombe tout cuit dans le bec »

Le beau-père : de même, le beau-père ne peut faire entendre sa parole : "Géraldine devrait être plus indépendante, elle attend toujours une instruction, sa mère est trop couveuse, je lui ai dit maintes fois qu'elle se lave seule ». Il ajoute : «Tu lui as essuyé les fesses il n’y a pas longtemps ; Géraldine attend toujours que sa mère fasse tout, qu'elle lui apporte une cuiller. La mère les maintient dans un cocon ».

La mère s'appuie sur les supposés besoins de sa fille : « elle a dormi avec nous ces quelques nuits, ça ne peut pas lui faire de mal. Les hommes disent que je la materne, elle a besoin que je l'habille, que je lui brosse les dents, c'est du temps que je passe avec elle, c'est plus du toucher, du contact »

Dans cette histoire qui se joue entre mère et fille, se dessine la jouissance du corps de Géraldine, manipulé par la mère comme celui d'un tout petit enfant. « Les hommes », rassemblés dans une formule qui marque l'indistinction, l'ont repéré, mais en pure perte semble-t-il car Mme G. ne fait pas cas de leur parole.

Le père ne réussit pas à se faire entendre, même chez lui, en l'absence de la mère. La mère semble occuper une position de toute-puissance, comme si elle était seule en cause dans ce qui arrive à sa fille. Le lien entre elle et sa fille n'apparaît en aucune façon médié par un tiers, père ou beau-père. En ce sens, Géraldine est posée du point de vue de la mère, en tant qu'objet de son désir, et la parole des hommes, père et beau-père, n'est pas entendue. À cet endroit, fait défaut la relation de la mère en tant que « ce qui en fait le caractère décisif est à isoler comme relation non pas au père, mais à la parole du père » (Lacan, 1998, 193) [19]. À propos du cas du « petit Hans » (Freud, 1909) [20], Lacan souligne l'ambiguïté de la mère et l'absence du troisième temps de l'œdipe, et notifie : « Il y a problème pour autant que la position du père est mise en question par le fait que ce n'est pas sa parole qui fait loi à la mère » (Lacan, 1998, 193) [19]. Ici, la mère apparait comme la seule qui sait au sujet de sa fille.

En conclusion, ce père souffre de l'emprise que la mère exerce sur Géraldine, du fait que sa parole reste nulle et non avenue, et de l'absence de reconnaissance de sa position de père, puisqu'il est interchangeable avec le beau-père, dans la parole maternelle.

\subsection{L'éloignement et la séparation : le père de Bertrand}

La mère vient en consultation pour son fils Bertrand, qui manifeste des comportements agressifs dans le cadre familial et scolaire. Les parents se sont séparés quatre mois auparavant. Suite à la séparation, Mme B. s'est « rapprochée de sa famille », pour vivre chez ses parents. Elle précise que c'est « pour Bertrand, pour qu'il profite de ses grands-parents »

Or, profiter des grands-parents, c'est s'éloigner du père de plusieurs centaines de kilomètres. Le père (et la mère) de la mère vient-il remplacer le père de Bertrand dans le fantasme maternel ? Les pères sont-ils interchangeables ? Dans le cas de Bertrand comme dans celui de Géraldine, ils n'occupent pas une position privilégiée à l'égard de l'enfant : père, père de la mère, beau-père comme dans le cas de Géraldine, tous se valent, ou plutôt certains semblent même être préférables. Or, il n'est pas de meilleur père, le père est (Viaux, 2002) [21].

Le père dit d'emblée : « Bertrand me manque énormément ». M. G. souffre de la séparation d'avec son fils, aggravée par l'éloignement - ils se voient peu. Cette souffrance vient en écho à celle de Bertrand qui revient du séjour chez son père en disant « je voulais pas repartir».

Il évoque aussi des "difficultés à parler » avec son fils « c'est pénible quelquefois, au lieu de dire ce qui ne va pas, il garde ça pour lui, parle des pokemons, du film qui est sorti » Pour tenter de trouver une explication, M. G. évoque son propre père qui « était solitaire ». Il ajoute que lui-même enfant était « très introverti » et qu'encore maintenant il aime s' « isoler ». Il ajoute sans transition que sa « mère est morte » de leucémie alors qu'il avait 11 ans. Il précise que son « père a fait ce qu'il a pu », puis confie qu'« au lieu de trouver les mots il préférait frapper » et qu'il n'était « pas patient ».

Le silence du grand-père paternel à l'origine, se décline ensuite suivant les générations : le grand-père, M. G., Bertrand. C'est du moins ce qu'il en est dit. Le silence a-t-il valeur de trait identificatoire au père ? Et la violence ? Nous entendons une répétition qui se joue de génération en génération. M.G. souffre du silence de son fils, mais au-delà, du silence et de la violence de son propre père, consécutif au décès de la mère. Les souffrances actuelles réactivent des douleurs anciennes qui appartiennent à l'histoire du père.

Quant au couple aujourd'hui désuni, M.G. fait le constat douloureux d'une vie «qui se dégradait progressivement ». À la question de savoir s'ils en ont parlé avant la rupture, Il répond qu'ils « ne se parlent pas spécialement» et qu'il «ne tient pas à se lancer dans des grandes conservations avec elle». Le silence se joue donc aussi dans les relations de couple.

Cependant M. G. précise qu'il met un point d'honneur à « être différent [de son père] vis-à-vis de Bertrand » et « qu'il ne soit pas un enfant battu ». Si être différent, c'est ne pas être battu, c'est logiquement une manière d'énoncer que luimême se considère comme enfant battu. L'expérience ayant montré certaines difficultés récurrentes des anciens enfants battus pour exercer une autorité sur leurs propres enfants, je l'interroge au sujet de cette différence qu'il a mentionnée. Il dit que «c'est la carotte avec Bertrand, malheureusement » et qu'il « préfère la carotte au fait de me fâcher ».

Il reconnaît la difficulté à exercer une autorité sur son fils, ce qu'il déplore en précisant « malheureusement ». Cette difficulté mainte fois évoquée lors des divorces, plutôt par les mères seules d'ailleurs, est souvent attribuée aux conséquences de la séparation parentale et au fait de se trouver seule avec l'enfant. Cette difficulté est aussi attribuée au fait que les mères n'auraient pas autant d'autorité que les pères. Nous saisissons dans le cas de Bertrand qu'il n'en est rien. L'exer- 
cice de l'autorité est entravé par l'histoire infantile du père dont le propre père utilisait la violence comme mode éducatif. Dès lors que M. G. refuse ce moyen, il lui faudrait inventer d'autres modalités éducatives. Or, pour l'instant, il se trouve désarmé, n'ayant pu résoudre ce problème qu'au moyen de la « carotte ».

En conclusion, M. G. souffre de la séparation d'avec son fils et de l'éloignement, liés aux modalités du divorce. Il souffre de la diffıculté d'échanges langagiers avec son fils, ce qui provient de son histoire infantile et de l'histoire des liens avec son épouse. Certes, la distance ne facilite évidemment pas ces échanges, mais tout n'est pas consécutif au divorce. Il éprouve des diffıcultés pour exercer son autorité, mais elles s'originent également dans sa propre histoire.

Ces deux exemples révèlent des éléments communs mais aussi singuliers dans la souffrance de ces pères. Ces deux illustrations cliniques proviennent d'un corpus de 11 cas qui ont fait l'objet d'une recherche exposée dans notre thèse portant sur l'absence du père lors des divorces. Nous en dégageons ici les conclusions relatives aux éléments généraux et singuliers présents dans les souffrances des pères lors des divorces, au cours de cette période spécifique entre 1970 et 2002, correspondant à deux lois majeures portant sur l'autorité parentale (Metz, 2003) [22].

\section{Conclusion}

L'institution du père a traversé des bouleversements inouïs depuis deux siècles. Le père, déchu du piédestal que lui conférait la puissance paternelle, invité à prendre la parole pour son enfant en 1970, s'est retrouvé aussitôt bâillonné par les transformations des modes de vie et les inadéquations du droit. Pendant les quelques 30 années qui ont suivi la loi sur l'autorité parentale, pris dans un entre-deux difficile, les pères ont perdu la puissance paternelle, sans pour autant être assurés de disposer de l'autorité parentale.

À l'aube du xxi siècle, l'institution du père a ainsi subi plusieurs séismes qui nécessitent d'être pris en considération. Cette institution qui tenait son sens dans le fait d'être une construction culturelle symbolique a perdu sa signification en trouvant un critère irréfutable dans la biologie. La mère a acquis une toute-puissance dans le fait de pouvoir contraindre ou exclure le père et c'est la perte de sens de l'institution du père qui donne ce pouvoir aux mères. Le droit, qui vient à peine de concéder un statut au père dans les familles non maritales, échoue à enrayer l'extinction des liens entre père et enfant lors des divorces et des séparations.

À la lumière de nos exemples cliniques, nous concluons que la défaillance du temps institutionnel au cours des dernières décennies s'est accompagnée d'une fragilisation du temps familial. Ainsi, cet affaiblissement du « forçage juridique » empêchant les confusions générationnelles (Legendre, 1985, 303) [15] a pu nuire à l'accès des hommes à la situation de père.

En ce qui concerne le temps familial, un homme devient père en fonction du sens de la paternité dans son histoire per- sonnelle, dans l'histoire de la femme qui l'a choisi comme père de son enfant, et en fonction de leurs liens réciproques autrefois désirants. Les enjeux psychiques conscients et inconscients liés au vécu infantile des parents se retrouvent impliqués dans les liens avec leurs enfants mais aussi dans les liens avec l'ancien conjoint. Ainsi, le divorce ou la séparation agit comme un révélateur d'éléments en puissance et ce qui se joue lors de la rupture est largement antérieur à la séparation.

La souffrance des pères lors des divorces contient donc des éléments généraux et des éléments singuliers que nos exemples cliniques ont tenté d'éclairer. De manière générale, ces pères que nous avons entendus souffrent des représentations issues de l'imaginaire collectif, liées à l'ère du paterfamilias, pourtant révolue. Ils souffrent également d'être disqualifiés et d'être soupçonnés (comme absents, mauvais pères, abuseurs pour ne citer que quelques motifs). Ils souffrent d'être évincés, et cette éviction au sein de la famille résonne avec leur éviction légale organisée dans nos sociétés, au profit d'un droit des mères exorbitantes, durant cette période de plus de 30 ans. Ce droit coïncide avec l'emprise maternelle au sein des familles, que nous soulignons dans le cas de Géraldine. Enfin, l'éviction familiale prend des formes variables singulières parmi lesquelles le cas de Géraldine révèle la souffrance du père face à la nullité de sa parole et à son interchangeabilité et celui de Bertrand révèle la souffrance de la séparation, de la difficulté de parler avec son fils et d'exercer une autorité

Les évolutions récentes du droit et de la société tentent de fournir à nouveau un pilier institutionnel aux sujets qui deviennent pères. En effet, au cours de la dernière décennie, le droit a lentement formulé de nouveaux fondements qui protègent le statut du père, et les pratiques familiales ont progressivement évolué dans le même sens. Les filiations paternelles sont beaucoup mieux assurées car les pères dans les familles naturelles reconnaissent majoritairement leurs enfants et car l'amélioration de la législation dans le cadre des procréations médicalement assistées protège désormais les filiations paternelles depuis la loi de 1994 (Art. 311-19, 311-20 du code civil).

\section{Références}

[1] Bruel A. Un avenir pour la paternité ? Rapport présenté le 24 juin 1997 au ministère de l'emploi et de la solidarité. s.l. Le Scribinaire; 1998.

[2] Hurstel F. La Déchirure paternelle. Paris: PUF; 1996 (rééd. 2002).

[3] Villeneuve-Gokalp C. La double famille des enfants de parents séparés. In: Population. Janvier 1999. p. 9 (1).

[4] Hurstel F, Hoffmann C. D'une absence à l'autre. Psychiatr Fr Décembre 1993;24(4):49-59.

[5] Metz C. L'enfant à l'épreuve de l'éloignement du père. Rev Psychiatr Psychol Med Novembre 1999;32:60-2.

[6] Chombeau C. Le développement des droits de l'enfant en France. Le Monde; 1993 (27 février : 12).

[7] Delumeau J, Roche D. Histoire des pères et de la paternité. Paris: Larousse; 2000.

[8] Lacan J. Le séminaire livre IV, la relation d'objet. Paris: Seuil; 1994. 
[9] Hurstel F, Delaisi De Parseval G. Le pardessus du soupçon. In Histoire des pères et de la paternité. Paris: Larousse; 2000. p. 381-99.

[10] [4 screens]. Available from : URL : http://www.insee.fr/FR/FFC/ DOCS_FFC/ip 624.pdf.

[11] Hurstel F. La fonction paternelle aujourd'hui en France : questions d'actualités et problèmes de théorie. Thèse d'Etat. Strasbourg I : Université Louis Pasteur; 1991.

[12] Villeneuve-Gokalp C. De la famille d'origine à la famille recomposée. In: Les recompositions familiales aujourd'hui. Paris: Nathan; 1993. p. $67-79$

[13] [4 screens]. Available from : URL : http://www.insee.fr/fr/ffc/docs ffc/IP825.pdf.

[14] Legendre P. Le crime du caporal Lortie. Traité sur le père. Paris: Fayard; 1989.
[15] Legendre P. L'inestimable objet de la transmission. Paris: Fayard; 1985 .

[16] Thévenot A. Les femmes dans le divorce en France aujourd'hui. Etude psychologique. Thèse. Strasbourg I : Université Louis Pasteur; 1993.

[17] Hurstel F. La famille après le divorce. La construction de la parenté dans les recompositions familiales. Dialogue 4e trimestre 1994;126: $73-81$.

[18] Lacan J. Séminaire R.S.I.1974-1975. notes dactylographiées. 21 janvier 1975.

[19] Lacan J. In: Le séminaire livre V, Les formations de l'inconscient. Paris: Seuil; 1998.

[20] Freud S. (1909) Cinq psychanalyses. Paris: PUF; 1990.

[21] Viaux J-L. (1997) L'enfant et le couple en crise. Du conflit psychologique au contentieux juridique. Paris: Dunod; 2002.

[22] Metz C. Absence du père et souffrance psychique lors des divorces et séparations. Thèse. Strasbourg I : Université Louis Pasteur; 2003. 